\title{
Carga em Presença de Folha Dielétrica e a Atração entre Elas
}

(A charge in front of a dielectric foil and the attraction between them)

\author{
G. F. Leal Ferreira \\ FCM-IFSC-USP, CP 369, 13560-970. São Carlos, SP \\ guilherm@ifsc.sc.usp.br
}

Recebido em 6 de junho, 2002. Aceito em 6 de agosto, 2002.

\begin{abstract}
Resolve-se o problema de uma carga pontual em presença de uma folha dielétrica fina, com o uso de imagens de cargas e dipolos cujos campos satisfazem as condições de continuidade do potencial e da componente normal do deslocamento elétrico nas superfícies da folha. Calcula-se, então, a força de atração resultante.
\end{abstract}

The problem consisting of a point charge placed in front of a thin dielectric foil is solved using images of charges and dipoles, which makes the continuity of the potential and of the normal component of the dielectric displacement to be satisfied at the plane boundaries of the foil. The attraction is then calculated.

\section{Introdução}

Seja como na Fig. 1 uma carga q a uma distância h do plano central de uma folha dielétrica fina, de espessura s e constante dielétrica K. Desejamos calcular a força de atração entre elas, mas para isso parece ser necessário resolver o problema por inteiro, isto é, achar o potencial em todos os pontos do espaço, desde que não é tarefa simples descobrir como são as distribuições de dipolo induzido na folha. Na Ref. 1, o problema de uma carga em presença de um bloco dielétrico de superfícies planas é tratado em geral, através de desenvolvimento do potencial em funções de Bessel. Análise desse tratamento analítico para o caso limite de uma folha (bloco pouco espesso, ver seção VII.1) sugere ser possível abordar o problema através do uso de imagens de cargas e dipolos, cujos campos, conjuntamente com os da carga indutora, satisfazem, em aproximação conveniente, as condições de continuidade do potencial e da componente normal do deslocamento elétrico nas superfícies da folha. Calcula-se então a força de atração. O campo elétrico no interior da folha é estudado na seção VII.2.

\section{Colocando o problema}

Na Fig. 1, a carga q está situada a uma distância h do plano médio (não mostrado) da folha, de espessura s, e constante dielétrica K. P é um ponto genérico da folha, distante $\mathrm{R}$ do ponto $\mathrm{O}^{\prime}$, projeção de q sobre o plano. $\theta$ é o ângulo entre $\mathrm{qP}$ e qO', que é o mesmo entre a normal PNe qP.

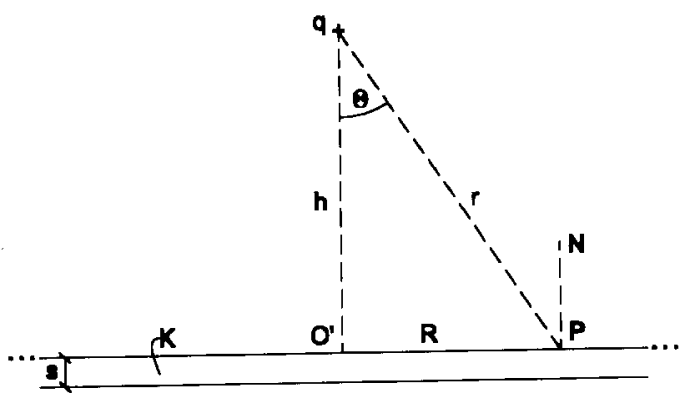

Figura 1. Carga q à distância $\mathrm{h}$ de folha fina dielétrica plana, de espessura s e constante dielétrica $\mathrm{K}$. O ponto $\mathrm{P}$ dista $\mathrm{R}$ de $\mathrm{O}$ ', projeção de q sobre a folha. PN é normal e $\theta$ é o ângulo entre $\mathrm{qP}$, igual a r, e $\mathrm{PN}$.

Para se obter a solução do problema por meio de imagens, consideraremos o espaço dividido em três regiões, I, II e III, como mostrado na Fig. 2. Nesta, a espessura da folha foi exagerada para incluir-se o seu plano médio em tracejado. Todas as imagens, quer de carga, quer de dipolo, estarão ou na posição de q ou na posição de sua imagem especular através do plano médio, abaixo da folha. A região I é a região acima da folha, Fig.2a. Nela agirão, além da carga q, uma carga imagem qI e um dipolo de momento $\mathrm{m}_{\mathrm{I}}$, orientado para cima e colocado abaixo da folha. Espera-se que qI se anule, visto que a reação da folha à presença de q é dipolar e já seria representada por $\mathrm{m}_{\mathrm{I}}$, que, aliás, deverá resultar negativo. A região II é o interior da folha, e 
será influenciada por carga qII e dipolo $\mathrm{m}_{\mathrm{II}}$ (orientado para baixo) na posição de q, e, na posição simétrica, por carga q'iı e dipolo m' II (orientado para cima), Fig.2b. Finalmente, a região III será o espaço abaixo da folha, influenciada por carga qIII e dipolo m $\mathrm{III}_{\text {(orientado }}$ para baixo), na posição de q. Vemos, portanto, que, por convenção, todas as cargas são assumidas positivamente e que os dipolos acima da folha estão orientados para baixo, e o oposto ocorrendo àqueles situados abaixo da folha. Esta convenção facilita os cálculos a seguir. Também porque a reação da folha é dipolar, espera-se que qIII resulte igual a q.

I

q •
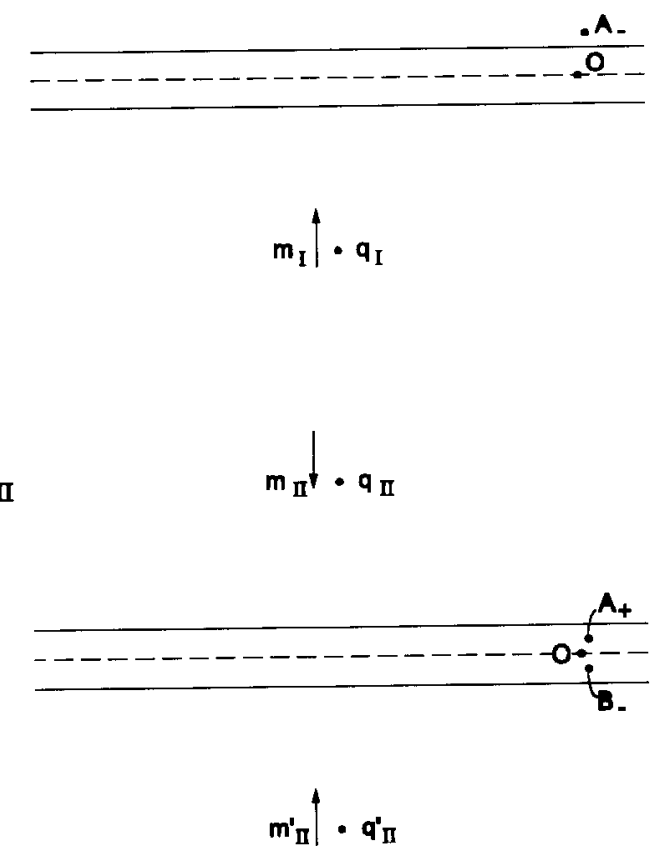

III
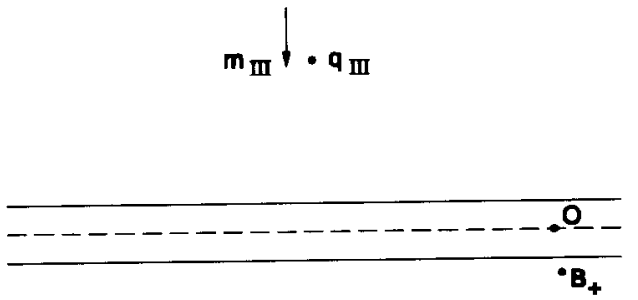

Figura 2. Divisão apropriada do espaço: a) região acima da folha, em que agem $q, q_{1}$, e $\left.m_{1} ; b\right)$ interior da folha, em que agem $q_{I I}, m_{I I}, q_{\text {II }}^{\prime}$ e $\left.m_{\text {II }}^{\prime} ; c\right)$ região abaixo da folha, agindo qII e miII. Em tracejado o plano médio da folha, onde está situado o ponto de referência $\mathrm{O}$. $\mathrm{A}_{-}$e $\mathrm{A}_{+}$, e $\mathrm{B}_{-}$e $\mathrm{B}_{+}$são pontos contíguos de cada lado das superfícies.

\section{Impondo as condições de contorno}

Temos oito incógnitas a determinar, quatro cargas e quatro momentos de dipolo. Para a determinação delas, vamos impor a continuidade do potencial e da componente normal do deslocamento elétrico nas superfícies da folha, Fig. 2. Sobre a superfície de cima, em $A_{-}$, região $\mathrm{I}$, agem $\mathrm{q}$, qI e $\mathrm{m}_{\mathrm{I}}$; e no ponto contíguo $\mathrm{A}_{+}$, já no interior da folha, agem qII, q' face de baixo, em $\mathrm{B}_{-}$, agem estas mesmas cargas e dipolos, e em $\mathrm{B}_{+}$, já fora da folha, a carga qIII e o dipolo miII. Então, em $\mathrm{A}_{-} \mathrm{A}_{+}$, , bem como em $\mathrm{B}_{-} \mathrm{B}_{+}$, vamos impor aquelas condições de contorno, mas com a precaução de tomar em conta a espessura da folha, ou seja, a da variação daquelas grandezas devido à variação da distância entre as fontes e os pontos tais como $\mathrm{A}_{ \pm}$e $\mathrm{B}_{ \pm}$. Para isso, tomaremos como referência o que ocorre para o plano médio, Fig.2, por exemplo o ponto $\mathrm{O}$ situado entre $\mathrm{A}_{ \pm}$e $\mathrm{B}_{ \pm}$, e calcularemos as grandezas nestes últimos pontos tendo em conta um deslocamento na vertical de s/2, para cima ou para baixo. Isto fará com que, por exemplo, no potencial das cargas naqueles pontos apareçam termos do tipo dipolo, que se misturarão aos termos de potencial puramente dipolares, os quais, no espírito da aproximação, não serão corrigidos pela variação de distância. Para a continuidade da componente normal do deslocamento (produto da constante dielétrica pelo campo elétrico) adotar-se-á o mesmo procedimento.

\section{Potenciais e campos, prepa- ratório}

Vamos exemplificar o que dissemos na seção anterior. Para a simplificação da escrita usaremos o sistema CGS, com a reversão ao MKS se fazendo pela divisão por $4 \pi \epsilon_{0}$. Tomemos, por exemplo, a carga qII e dipolo $\mathrm{m}_{\mathrm{II}}$, Fig.2b, e escrevamos os potenciais $\mathrm{V}_{\mathrm{qII}}\left(\mathrm{A}_{+}\right)$e $\mathrm{V}_{\mathrm{mII}}\left(\mathrm{A}_{+}\right)$ que criam em $\mathrm{A}_{+}$. Eles são

$$
\begin{gathered}
V_{q I I}\left(A_{+}\right)=V_{q I I}(O)+\nabla V_{q I I}(O) \cdot \overrightarrow{O A}=\frac{q}{r}+\frac{q s \cos \theta}{2 r^{2}} \\
V_{m I I}\left(A_{+}\right) \simeq V_{m I I}(O)=\frac{m_{I I} \cos \theta}{r^{2}}
\end{gathered}
$$

lembrando que a espessura da folha é s e que r é contado da fonte (carga ou dipolo) até O. Para o potencial no ponto B_ o segundo termo do lado direito da Eq.1 será negativo enquanto que a Eq.2 permanecerá válida.

Para o cálculo da componente normal do campo elétrico, necessário para a especificação do deslocamento) vamos escrever primeiro o campo elétrico $\vec{E}_{q I I}(T)$ numa vizinhança $\mathrm{T}$ de $\mathrm{O}$ 


$$
\vec{E}_{q I I}(T)=\vec{E}_{q I I}(O)+\frac{q}{r^{3}}\left[\left(1-3 \operatorname{sen}^{2} \theta\right) \overrightarrow{d R}+\left(1-3 \cos ^{2} \theta\right)(-\hat{n}) d p\right]
$$

sendo

$$
\overrightarrow{O T}=\overrightarrow{d R}+(-\hat{n}) d p
$$

estando $\overrightarrow{d R}$ na direção radial de O' a $\mathrm{O}$ e $\hat{n}$ no sentido de PN, Fig. 1. dp é um diferencial de distância. Então, a componente do campo $\mathrm{E}_{\mathrm{qIIn}}\left(\mathrm{A}_{-}\right)$de qII em $\mathrm{A}_{-}$, na direção de - $\hat{n}$, e com $\mathrm{dp}=-\mathrm{s} / 2$ será

$$
E_{q I I n}\left(A_{-}\right)=E_{q I I n}(O)-\frac{q s\left(1-3 \cos ^{2} \theta\right)}{2 r^{3}}
$$

Para o dipolo de momento $\mathrm{m}_{\mathrm{II}}$, o seu campo normal $\mathrm{E}_{\mathrm{mIIn}}\left(\mathrm{A}_{-}\right)$em $\mathrm{A}_{-}$, na direção -⿳亠丷⿵冂丶 , será

$$
E_{m I I n}\left(A_{-}\right) \simeq E_{m I I n}(O)=-\frac{m_{I I}\left(1-3 \cos ^{2} \theta\right)}{r^{3}}
$$

\section{Os potenciais e os desloca- mentos nas superfícies da fo- lha}

Damos, a seguir, os potenciais e as componentes normais $\left(D_{n}\right.$, com a convenção de ser positiva para baixo, - $\hat{\mathrm{n}}$ ) do deslocamento elétrico em $\mathrm{A}_{-}, \mathrm{A}_{+}, \mathrm{B}_{-}$e $\mathrm{B}_{+}$. Pelas convenções adotadas, os potenciais em $\mathrm{O}$ devido às cargas e os dipolos (Fig.2) são positivos. As Eqs.1, 2 e 4 serão usadas a seguir.

Em A_:

$$
\begin{aligned}
V_{q}\left(A_{-}\right)=\frac{q}{r}+\frac{q s \cos \theta}{2 r^{2}} \quad, \quad D_{q n}\left(A_{-}\right)=\frac{q \cos \theta}{r^{2}}-\frac{q s\left(1-3 \cos ^{2} \theta\right)}{2 r^{3}} \\
V_{q I}\left(A_{-}\right)=\frac{q_{I}}{r}-\frac{q_{I} s \cos \theta}{2 r^{2}} \quad, \quad D_{q I n}\left(A_{-}\right)=-\frac{q_{I} \cos \theta}{r^{2}}+\frac{q_{I} s\left(1-3 \cos ^{2} \theta\right)}{2 r^{3}} \\
V_{m I}\left(A_{-}\right)=\frac{m_{I} \cos \theta}{r^{2}} \quad, \quad D_{m I n}\left(A_{-}\right)=\frac{m_{I}\left(1-3 \cos ^{2} \theta\right)}{r^{3}}
\end{aligned}
$$

$\mathrm{Em} \mathrm{A}_{+}:$

$$
\begin{gathered}
V_{q I I}\left(A_{+}\right)=\frac{q_{I I}}{r}+\frac{q_{I I} s \cos \theta}{2 r^{2}} \quad, \quad D_{q I I n}\left(A_{+}\right)=K\left[\frac{q_{I I} \cos \theta}{r^{2}}-\frac{q_{I I} s\left(1-3 \cos ^{2} \theta\right)}{2 r^{3}}\right] \\
V_{m I I}\left(A_{+}\right)=\frac{m_{I I} \cos \theta}{r^{2}} \quad, \quad D_{m I I n}\left(A_{+}\right)=-K \frac{m\left(1-3 \cos ^{2} \theta\right)}{r^{3}} \\
V_{q \prime I I}\left(A_{+}\right)=\frac{q_{I I}}{r}-\frac{q_{I I} s \cos \theta}{2 r^{2}} \quad, \quad D_{q I I n}\left(A_{+}\right)=K\left[-\frac{q_{I I} \cos \theta}{r^{2}}+\frac{q_{I I} s\left(1-3 \cos ^{2} \theta\right)}{2 r^{3}}\right] \\
V_{m \prime I I}\left(A_{+}\right)=\frac{m_{I I} \cos \theta}{r^{2}} \quad, \quad D_{m I I I n}\left(A_{+}\right)=K \frac{m_{I I}\left(1-3 \cos ^{2} \theta\right)}{r^{3}}
\end{gathered}
$$

Em B-:

$$
\begin{gathered}
V_{q I I}\left(B_{-}\right)=\frac{q_{I I}}{r}-\frac{q_{I I} s \cos \theta}{2 r^{2}} \quad, \quad D_{q I I n}\left(B_{-}\right)=K\left[\frac{q_{I I} \cos \theta}{r^{2}}+\frac{q_{I I} s\left(1-3 \cos ^{2} \theta\right)}{2 r^{3}}\right] \\
V_{m I I}\left(B_{-}\right)=\frac{m_{I I} \cos \theta}{r^{2}} \quad, \quad D_{m I I n}\left(B_{-}\right)=-K \frac{m_{I I}\left(1-3 \cos ^{2} \theta\right)}{r^{3}} \\
V_{q \prime I I}\left(B_{-}\right)=\frac{q_{I I}^{\prime}}{r}+\frac{q_{I I} s \cos \theta}{r^{2}} \quad, \quad D_{q I I n}\left(B_{-}\right)=K\left[-\frac{q_{I I} \cos \theta}{r^{2}}-\frac{q_{I I} s\left(1-3 \cos ^{2} \theta\right)}{2 r^{3}}\right] \\
V_{m \prime I I}\left(B_{-}\right)=\frac{m_{I I} \cos \theta}{r^{2}} \quad, \quad D_{m \prime I I n}\left(B_{-}\right)=K \frac{m_{I I}\left(1-3 \cos ^{2} \theta\right)}{r^{3}}
\end{gathered}
$$

$\mathrm{Em} \mathrm{B}_{+}$:

$$
\begin{array}{cc}
V_{q I I I}\left(B_{+}\right)=\frac{q_{I I I}}{r}-\frac{q_{I I I} s \cos \theta}{2 r^{2}} \quad, \quad D_{q I I I n}\left(B_{+}\right)=\frac{q_{I I I} \cos \theta}{r^{2}}+\frac{q_{I I I} s\left(1-3 \cos ^{2} \theta\right)}{2 r^{3}} \\
V_{m I I I}\left(B_{+}\right)=\frac{m_{I I I} \cos \theta}{r^{2}} \quad, \quad D_{m I I I n}\left(B_{+}\right)=-\frac{m_{I I I}\left(1-3 \cos ^{2} \theta\right)}{r^{3}}
\end{array}
$$




\section{Impondo as condições de contorno}

Impomos, agora, as condições de contorno ao potencial e à componente normal do deslocamento nas duas superfícies da folha. Ou seja

$$
\begin{gathered}
V_{q}\left(A_{-}\right)+V_{q I}\left(A_{-}\right)+V_{m I}\left(A_{-}\right)=V_{q I I}\left(A_{+}\right)+V_{m I I}\left(A_{+}\right)+V_{q I I I}\left(A_{+}\right)+V_{m I I I}\left(A_{+}\right) \\
D_{q n}\left(A_{-}\right)+D_{q I n}\left(A_{-}\right)+D_{m I n}\left(A_{-}\right)\left(A_{-}\right)=D_{q I I n}\left(A_{+}\right)+D_{m I I n}\left(A_{+}\right)+D_{q I I n}\left(A_{+}\right)+D_{m I I I N}\left(A_{+}\right) \\
V_{q I I}\left(B_{-}\right)+V_{m I I}\left(B_{-}\right)+V_{q I I I}\left(B_{-}\right)+V_{m I I I}\left(B_{-}\right)=V_{q I I I}\left(B_{+}\right)+V_{m I I I}\left(B_{+}\right) \\
D_{q I I n}\left(B_{-}\right)+D_{m I I n}\left(B_{-}\right)+D_{q I I n}\left(B_{-}\right)+D_{m I I n}\left(B_{-}\right)=D_{q I I I n}\left(B_{+}\right)+D_{m I I I n}\left(B_{+}\right)
\end{gathered}
$$

Substituímos, nestas equações, os valores dados nas Eqs.7-19 e separamos, em cada uma delas, os termos de carga e dipolo, obtendo-se oito equações que determinarão as oito incógnitas. As equações de carga levam imediatamente a

$$
q_{I}=0, q_{I I}=\frac{q}{2 K}(K+1), q_{I I}^{\prime}=\frac{q}{2 K}(K-1), q_{I I I}=q
$$

Para os termos dipolares obtém-se

$$
\begin{gathered}
\left(q+q_{I}\right) \frac{s}{2}+m_{I}=\left(q_{I I}-q^{\prime} I I\right) \frac{s}{2}+m_{I I}+m_{I I} \\
\left(-q+q_{I}\right) \frac{s}{2}+m_{I}=K\left[\left(q^{\prime}{ }_{I I}-q_{I I}\right) \frac{s}{2}+m_{I I}-m_{I I}\right] \\
\left(-q_{I I}+q^{\prime} I I\right) \frac{s}{2}+m_{I I}+m_{I I}=-q_{I I I} \frac{s}{2}+m_{I I I} \\
K\left[\left(q_{I I}-q^{\prime} I I\right) \frac{s}{2}-m_{I I}+m_{I I I}\right]=q_{I I I} \frac{s}{2}-m_{I I I}
\end{gathered}
$$
dipolo

Das equações 25-28, usando-se os resultados na Eq.24, os seguintes valores são obtidos para os momentos de

$$
m_{I}=-\frac{(K-1) q s}{2 K}, m_{I I}=\frac{(K-1) q s}{4 K^{2}}, m_{I I}=-m_{I I}, m_{I I I}=-m_{I}
$$

\section{A força de atração entre a carga e a folha}

A força de atração entre a folha e a carga será igual à força entre $m_{I}$ e q. Com a convenção da Fig.2a para a orientação de $\mathrm{m}_{\mathrm{I}}$, e tendo em conta que este é negativo (Eq.29), resultará força de atração F sobre a carga $(\mathrm{r}=2 \mathrm{~h})$, normal à folha, e igual a

$$
F=\frac{(K-1) q^{2} s}{8 K h^{3}}
$$

ou no MKS

$$
F=\frac{(K-1) q^{2} s}{32 \pi \epsilon_{0} K h^{3}}
$$

\section{Discussão}

\section{VII.1 Os valores obtidos para as cargas e dipolos imagens}

Como previsto na seção III, obteve-se $q_{I}=0$ e $q_{I I I}=q$ nas Eqs. 24. Quanto aos momentos de dipolo induzidos, obteve-se $\mathrm{m}_{\mathrm{II}}=-\mathrm{m}_{\mathrm{II}}$ e $\mathrm{m}_{\mathrm{III}}=-\mathrm{m}_{\mathrm{I}}$. Pelo menos esta última igualdade era de se esperar ocorrer, visto que a folha deve estar polarizada para baixo pelo campo de q. Essa distribuição normal de dipolos cria potencial negativo acima ( $\mathrm{m}_{\mathrm{II}}$ negativo) e positivo ( $\mathrm{m}_{\mathrm{III}}$ positivo) abaixo da folha, necessariamente de mesmo módulo. Notemos que todas as cargas e momentos de dipolo são finitos para $\mathrm{K} \rightarrow \infty$.

Como dissemos na Introdução, na Ref.1 resolve-se o problema de carga em presença de bloco dielétrico espesso com superfícies planas, desenvolvendo-se os potenciais na função de Bessel $\mathrm{J}_{0}$. Calcula-se explicitamente o potencial na região III, Fig. 2c, $\mathrm{V}_{\mathrm{III}}$, resultando em coordenadas cilíndricas $\rho$ e z, contadas das carga

$$
V_{I I I}=q\left(1-\beta^{2}\right) \int_{0}^{\infty} \frac{J_{0}(k \rho) e^{-k z} d k}{1-\beta^{2} e^{-2 k s}}, \quad \beta=\frac{K-1}{K+1}
$$


que para s pequeno, através do desenvolvimento em série $\mathrm{e}^{-2 \mathrm{ks}} \simeq 1-2 \mathrm{ks}$, aparentemente válido, leva a

$$
V_{I I I} \simeq q \int_{0}^{\infty} J_{0}(k \rho)\left[1-\frac{(K-1)^{2}}{2 K} k s\right] e^{-k z} d k=q\left[1+s \frac{(K-1)^{2}}{2 K} \frac{\partial}{\partial z}\right] \int_{0}^{\infty} J_{0}(k \rho) e^{-k z} d z
$$

com o termo de carga q e o de dipolo com momento $\mathrm{qs}(\mathrm{K}-1)^{2} / 2 \mathrm{~K}$, tendo em vista que a integral à direita da Eq.33 é igual a 1/r (o que nos sugeriu o uso de imagens de cargas e dipolos). Este termo de dipolo não concorda com o calculado na Eq.29, com o inconveniente de divergir para $K \rightarrow \infty$. Não sabemos a razão da discrepância.

\section{VII.2 O campo elétrico no interior da fo- lha}

O campo elétrico no interior da folha pode ser obtido dos resultados anteriores. Notemos primeiro que, sendo ele originário de cargas e dipolos situados fora dela, Fig.2b, o campo elétrico tem aí divergência nula. Então, não haverá densidade volumétrica de carga de polarização e esta se manifestará através de cargas superficiais. Chamando-se de y a distância vertical de um ponto próximo a $\mathrm{O}$, contada positivamente para baixo, a componente normal do campo elétrico $\mathrm{E}_{\mathrm{IIn}}(\mathrm{R}, \mathrm{y})$ é achada como sendo

$$
E_{I I n}(R, y)=\frac{q \cos \theta}{K r^{2}}+\frac{q\left(1-3 \cos ^{2} \theta\right) y}{K r^{3}}-\frac{q s(K-1)\left(1-3 \cos ^{2} \theta\right)}{2 K^{2} r^{3}}
$$

e para a componente paralela às faces da folha, $\mathrm{E}_{\mathrm{IIp}}$,

$$
E_{I I p}(R, y)=\frac{q \operatorname{sen} \theta}{r^{2}}-\frac{3 q y \operatorname{sen} \theta \cos \theta}{r^{3}}
$$

Não é difícil verificar que o primeiro termo no lado direito da Eq. 34 é a componente normal do campo resultante de q e das densidades de carga de polarização vizinhas. O segundo termo, de mesma dependência na constante dielétrica que o primeiro, deve advir de correção ao primeiro em razão da espessura finita da folha. Já o terceiro no lado direito da Eq.34 e anulandose para $K=1$ deve ser atribuído à polarização (normal) não uniforme na folha, devida ao primeiro termo de $\mathrm{E}_{\mathrm{IIn}}(\mathrm{R}, \mathrm{y})$. Com relação à componente paralela, Eq.35, ela é devida exclusivamente à carga q e, sendo independente de $\mathrm{K}$, a polarização por ela gerada não age diretamente sobre a carga q. Este resultado concorda com a previsão de que só a distribuição de dipolos normal à folha seria ativa.

\section{Considerações finais e agra- decimentos}

Certa vez, o Prof. Paulo Cesar de Camargo, da Universidade Federal do Paraná, comentou que muitas experiências interessantes em Eletrostática podem ser feitas explorando-se a atração entre cargas e folhas dielétricas, mas que a literatura não não faz referência a essa situação. A ele, pois, dedico o cálculo presente. $\mathrm{O}$ autor agradece bolsa de produtividade do CNPq.

\section{Referência}

1. W. R. Smythe, Static and Dynamic Electricity, McGraw-Hill Book Co., N. York (1950), Cap. 5. 